\title{
Mechanisms for sustainable post-trial access: A perspective
}

\author{
P Naidoo, ${ }^{1}$ MB BCh, MMedSci (Pharmacol), BPharm (Hons); V Rambiritch, ${ }^{2}$ PhD; D A Webb, ${ }^{3}$ BSc (Hons), MB BCh; \\ R F Leisegang, ${ }^{4}$ BSC Eng (Honours), MB ChB, PhD; M F Cotton, ${ }^{5}$ MB ChB, MMed (Paed), FCPaed (SA), PhD, DCH (SA), DTM\&H (SA); \\ H R Etheredge, ${ }^{6} \mathrm{PhD}$ \\ ${ }^{1}$ Department of Internal Medicine, King Edward VIII Tertiary Hospital, Umbilo, South Africa \\ ${ }^{2}$ Biomedical Research and Ethics Committee, University of KwaZulu-Natal, Durban, South Africa \\ ${ }^{3}$ Houghton House Addiction and Mental Health Treatment Centres, Johannesburg, South Africa \\ ${ }^{4}$ Department of Pharmaceutical Biosciences, Uppsala University, Sweden \\ ${ }^{5}$ Department of Paediatrics and Child Health, Faculty of Medicine and Health Sciences, Stellenbosch University, Cape Town, South Africa \\ ${ }^{6}$ Wits Donald Gordon Medical Centre, and Department of Internal Medicine, Faculty of Health Sciences, University of the Witwatersrand, Johannesburg, \\ South Africa
}

Corresponding author: P Naidoo (poobalan1naidoo@yahoo.com)

Clinical trials are essential to establish the safety and efficacy of investigational products, contributing to risk/benefit assessments that ultimately determine whether these products meet the criteria for market authorisation. Clinical trials are also an important source of revenue and expertise generation for countries in which they are conducted. In developing countries, they represent substantial foreign direct investment. In spite of the substantial capital input that clinical trials require, the issue of funding post-trial access to beneficial therapies remains contentious, especially in resource-limited settings. In this article, we explore this situation and propose mechanisms to establish 'win-win' situations where both patients and clinical trial sponsors derive benefit from post-trial access programmes in low- and middle-income countries.

S Afr J Bioethics Law 2021;14(3):77-78. https://doi.org/10.7196/SAJBL.2021.v14i3.782

Robust clinical trial programmes are a regulatory requirement and inform evidence-based medicine. Moreover, when no effective therapies exist, clinical trials may provide patients with access to potentially beneficial investigational therapies. At a macroeconomic level, clinical trials contribute to the host country's economy by creating employment. There is little doubting the many positive benefits associated with a clinical trial programme. However, with the large number of multinational trials being conducted in low- and middle-income countries (LMICS), post-trial care and post-trial access (PTA) to beneficial therapies is a contentious issue. ${ }^{[1]}$

Despite the Declaration of Helsinki support of PTA, ${ }^{[2]}$ there remains uncertainty and a lack of consensus ${ }^{[3]}$ about financial and operational mechanisms to ensure sustainable PTA.

Our aim is to outline potential mechanisms to ensure sustainable PTA. Although we focus on LMICs, PTA is also applicable to high-income countries (HICS) with unequal distribution of healthcare benefits. ${ }^{[4]}$ We limit our discussion to PTA because the provision of post-trial care is complex, and will be discussed in a subsequent publication.

\section{What happens when the trial ends?}

In resource-limited settings, the transition from the clinical trial experience to routine clinical care is often unsettling for participants: the beneficial intervention is withdrawn, and the patient is unlikely to be afforded the same monitoring and care that was the norm during the trial. Consequently, ongoing patient care may be compromised, and the conflict of interest and dual role of the clinician as trialist and caregiver become apparent. In our opinion, the negative biological and psychological consequences to the patient may be significant.
In cases where there is no alternative therapy and the investigational therapy receives market approval shortly after the trial, it may only be available at premium prices in these resource-limited settings. Even though patients contribute their time and carry potential risk during development of the therapy, cost barriers may limit their access to beneficial treatments on completion of the trial. This amounts to an unfair distribution of the benefits of the therapy among study subjects and clinical trial stakeholders, ${ }^{[5]}$ where the former are prejudiced in favour of the latter. This is incongruent with the core ethical principles of justice, autonomy and beneficence. The participants and clinicians may consider this subsequent lack of access as exploitation, with participant dignity being violated. ${ }^{[6]}$

In other instances, sponsors of clinical trials choose not to register a drug in the country where the clinical study was done. Under these circumstances, drug access requires a special import licence and incurs additional time, costs and administrative burdens for both patients and clinicians.

\section{Provision of PTA - what are the benefits?}

By ensuring uninterrupted access to effective and innovative treatment, PTA is respectful of basic human rights and patient dignity. Furthermore, it empowers the clinician to continue to act in the best interests of his or her patient without interference from an ongoing responsibility to the trial sponsor. It helps maintain patients' trust and confidence in both clinician and healthcare system. PTA also furthers the ends of justice, ensuring that those who have volunteered realise a long-term benefit to doing so. 
Pharmaceutical companies could equally be recognised for ensuring PTA within their corporate social responsibility programmes, thereby improving their image and investor confidence. The goodwill demonstrated by providing PTA may strengthen the relationship between the pharmaceutical industry and clinicians, patient advocate groups, health authorities, medical funders and universities.

The promise of PTA might incentivise patients to participate in studies, potentially shortening recruitment times, improving patient enrolment and limiting study drop-outs and loss to follow-up. This has the potential to reduce research and development costs and shorten the time required to bring revenue-generating innovative therapies to market. However, this may be perceived by some as a perverse incentive.

PTA programmes have the potential to supplement data collected during the clinical trial programme by generating critical longterm 'real-world' data on efficacy, harm, tolerability and quality of life..$^{[7]}$ Cost-effective data collection would be facilitated by using applications on patients' and clinicians' phones or case report forms integrated into existing electronic health record systems. It must be noted that certain LMICs may not have access to such applications.

Finally, universities with teaching hospitals are well placed to assist with post-trial data collection, analysis and dissemination at lower cost, given their research and ethics infrastructure. This would also serve to increase the universities' research and higher degree output.

\section{How do we make PTA affordable and sustainable?}

At the core of the sustainability of any PTA programme is how these programmes should be funded, and for how long this can be sustained. Society needs innovative therapies to address unmet medical needs, and therefore, given that the responsibility for the execution of clinical trials does not lie solely with the pharmaceutical industry, and that the benefits arising from them are far-reaching, finding solutions to PTA requires a combined effort from multiple stakeholders. These include study participants, clinicians, pharmaceutical companies, health authorities, clinical research organisations, medical funders and universities. Considerations relating to PTA include the substantial costs of producing increasingly precise, efficacious, tolerable and safe biological therapies for chronic diseases. As there is also significant shareholder pressure to maximise return on investment, providing PTA may not, prima facie, appear commercially appetising. One method currently employed to ensure PTA at the end of phase III studies is the enrolment of patients in an ongoing open-label extension study. Such roll-over studies are of relatively short duration and are primarily designed to address the gap between phase III completion and market authorisation in (HICs). In resource-limited settings, long registration times are common, and the roll-over study is likely to end before commercial availability, creating a treatment gap. This situation poses a threat to distributive justice, with participants in HICs likely enjoying far better PTA than their LMIC counterparts. Although roll-over studies are valuable in that they benefit patients, they fall short of providing the care needed, especially where a trial participant has assumed a substantial risk to bring the drug to market.

It must be said that, for at least some patients, PTA may not be necessary for their entire lifespan. Some individuals may recover or achieve remission, others may withdraw informed consent, better alternative treatments (e.g. with improved efficacy, tolerability, ease of use) may become available and patients may consent to entering other clinical trials that preclude use of the previous intervention. There is often a subset of patients that does not sufficiently respond to treatment to make ongoing treatment clinically worthwhile, and who may discontinue therapy with no biological ill effects. In essence, there is likely to be attrition, reducing the number of patients eligible for PTA and therefore also costs of ongoing supply of medicines.

Funding may be sourced from the pharmaceutical industry and included in the initial clinical trial budget. Specifically, these funds may be derived from funds allocated to corporate social responsibility projects, surplus clinical trial funds and medical affairs budgets for generation of real-world data. In addition, perhaps pharmaceutical companies benefitting from the sale of generic medicines, particularly in LMIC settings, where these generic manufacturers often dominate market share, should contribute financially to PTA. Governments benefit financially from clinical trials (e.g. through creation of employment), and could support PTA through tax incentives, rebates and reductions for companies providing PTA. Finally, an international fund could be established, providing philanthropists and clinical investigators an opportunity to contribute.

\section{Conclusion}

Robust clinical trials are necessary to generate the body of evidence required for safe and effective use of novel therapies, thereby supporting the concept of evidence-based medicine. In our opinion, clinicians and trial sponsors have a moral obligation to advocate for patients and take steps to ensure that beneficial therapies remain available to research participants who have given their time and risked potential biopsychological harm.

A co-ordinated multi-stakeholder paradigm shift is required to positively embrace and facilitate sustainable PTA. Detailed operational aspects to make the PTA programme successful may be best crafted after a pilot PTA programme, with subsequent iterations based on failures and successes, and ultimately the creation of a financial and legal framework.

Acknowledgements. None.

Author contributions. Equal contributions.

Funding. None.

Conflicts of interest. None.

1. Sofaer N, Strech D. Reasons why post-trial access to trial drugs should, or need not be ensured to research participants: a systematic review. Public Health Ethics 2011;4(2):160-184. https://doi.org/10.1093\%2Fphe\%2Fphr013

2. WMA Declaration of Helsinki - ethical principles for medical research involving human subjects. https://www.wma.net/policies-post/wma-declaration-ofhelsinki-ethical-principles-for-medical-research-involving-human-subjects/ (accessed 1 December 2021).

3. CookK, Snyder J, Calvert J. Attitudes toward post-trial access to medical interventions: A review of academic literature, legislation, and international guidelines. Dev World Bioeth 2016;16(2):70-79. https://doi.org/10.1111/dewb.12087

4. Grady $C$. The challenge of assuring continued post-trial access to beneficial treatment. Yale J Health Policy Law Ethics 2005;5(1):425-435

5. Ballantyne AJ. How to do research fairly in an unjust world. Am J Bioeth 2010;10(6):26-35. https://doi.org/10.1080/15265161.2010.482629

6. Andanda P, Wathuta J. Human dignity as a basis for providing post-trial access to healthcare for research participants: A South African perspective. Med Health Care Philos 2018;21(1):139-155. https://doi.org/10.1007/s11019-0179782-1

7. Katkade VB, Sanders KN, Zou KH. Real world data: An opportunity to supplement existing evidence for the use of long-established medicines in health care decision making. J Multidiscip Healthcare 2018;11:295-304. https://doi.org/10.2147/jmdh. s160029

Accepted 19 November 2021 